\title{
Versorgung in Zahlen
}

\author{
QUANTITATIVE VERSORGUNGSFORSCHUNG In Deutschland erhält \\ ein Viertel der Frauen mit einem Armlymphödem keine Lymphdrai- \\ nage. Lediglich der Hälfte aller Menschen mit Fibromyalgie verordnen \\ Ärzte aktive Maßnahmen. Und nur etwa 15 Prozent aller Menschen \\ mit Rheumatoider Arthritis bekommen Ergotherapie. All diese Fakten \\ wären ohne quantitative Versorgungsforschung nicht bekannt.
}

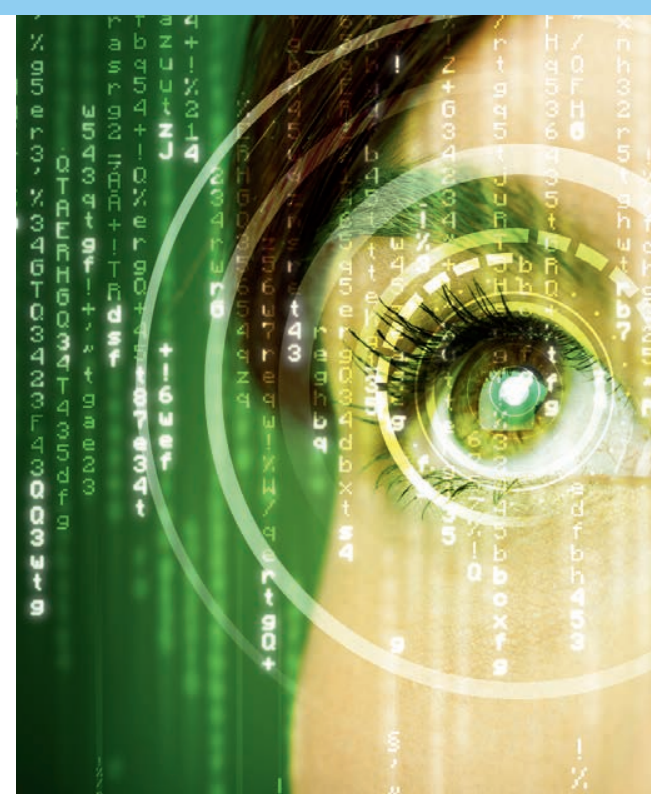

mitteln und um 17 Prozent bei den Hilfsmitteln $[4,5]$. Damit sie die Verordnungs- und Ausgabenentwicklung für Heilmittel verfolgen können, haben die Spitzenverbände der GKV ein Heilmittel-Informations-System (HIS) aufgebaut $[4,6]$.

Auch einzelne Krankenkassen fördern wissenschaftliche Analysen ihrer Daten, im Bereich der Heil- und Hilfsmittel etwa die BARMER GEK. Die Ergebnisse der Versorgungsforscher werden im jährlich erscheinenden BARMER GEK Heil- und Hilfsmittelreport publiziert. Hinsichtlich der Versorgung mit Heilmitteln können die Autoren allerdings nur Aussagen darüber treffen, welche Maßnahmen Ärzte auswählen. Welche Therapiemethoden eine Ergotherapeutin in die Behandlung hat einfließen lassen, bleibt unklar. Zudem ist es oftmals schwierig, Diagnose und Verordnung in einen Zusammenhang zu bringen unter anderem dann, wenn Folgeverordnungen ohne direkten Arztkontakt erfolgen oder ein Patient mehrere Diagnosen hat [2].

Wirksame Therapiemethoden zu selten verordnet > Die Versorgungsforscher analysieren für den BARMER GEK Heil- und Hilfsmittelreport nicht nur Abrechnungsdaten und Diagnosen, sondern ermitteln auch die Datenlage zur Wirksamkeit der Therapiemethoden. Dadurch fanden sie in den letzten Jahren heraus, dass es Methoden gibt, die trotz gesicherter oder vielversprechender Evidenz bei Verordnungen teilweise nicht genügend gaben dafür stiegen im Verhältnis deutlich stärker als in anderen Bereichen: zwischen 2007 und 2012 um 26,7 Prozent bei den Heil-
Krankenkassen unterstützen Versorgungsforschung > Heil- und Hilfsmittel sind in den letzten Jahren zunehmend in den Fokus von Versorgungsanalysen geraten. Denn die AusUm diese Ziele zu erreichen, haben Versorgungsforscher ein breites Spektrum an MögDaten auswerten, die sie zum Beispiel anhand von Fragebögen erheben. Oder sie analysieren
sogenannte Routinedaten, zu denen Daten der Routinedaten auswerten $>$ Die Routin lanten und stationären Versorgung, zur Arbeitsunfähigkeit und zur Arznei-, Heil- und Hilfsmittelversorgung [3]. In erster Linie diehelfen auch Versorgungsforschern. Diese könes möglich, Aussagen dazu zu treffen, welche Patienten mit welcher Erkrankung welche Maßnahmen in Anspruch nehmen [4]. 


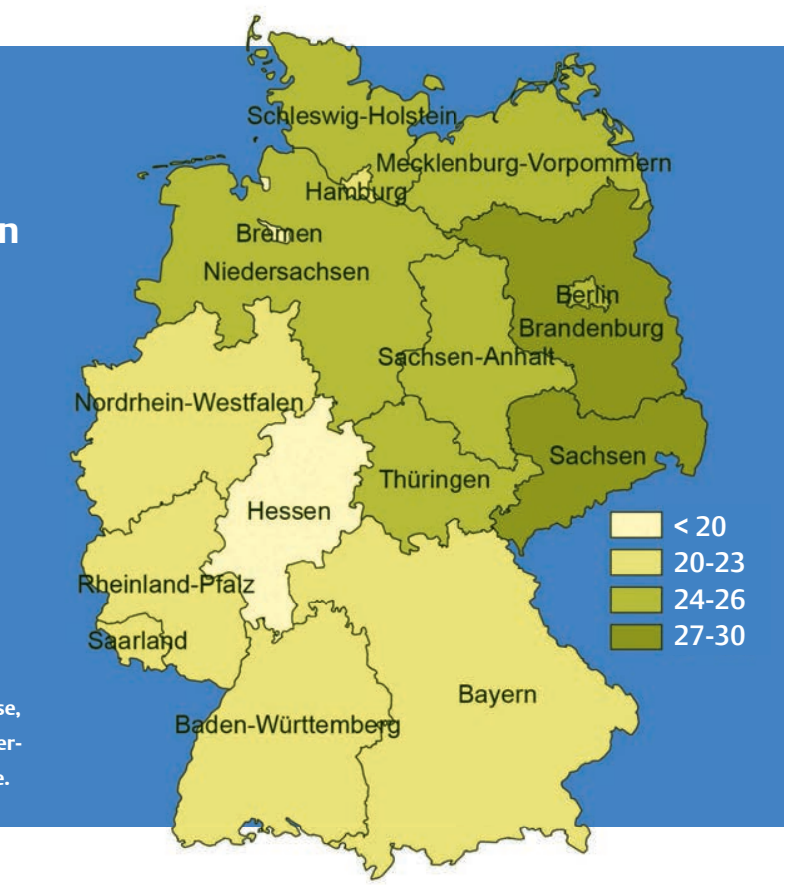

Für die Berechnungen zur Indikation wurden folgende Erkrankungen berücksichtigt: Schädel-Hirn-Trauma, Enzephalitis, zerebrale Blutung, zerebraler Tumor, Insult, Zerebralparese, Parkinson, Multiple Sklerose, ALS, Querschnittssyndrom und Polyneuropathie.

berücksichtigt werden [5]. Leidtragende waren zum Beispiel Versicherte der BARMER GEK mit Rheumatoider Arthritis und Lungenemphysem (COPD). Und das, obwohl Studien im Rahmen der Ergotherapie eine starke Evidenz für die Effektivität von Instruktionen zum Gelenkschutz und zum effizienten Funktionsgebrauch belegen. Darüber hinaus erwies sich die Schienenversorgung für Menschen mit Rheumatoider Arthritis als effektiv in der Schmerzreduktion und zur Steigerung der Handkraft. Bei der interdisziplinären Zusammenarbeit in der Behandlung von Patienten mit COPD wird die Ergotherapie noch nicht einmal erwähnt. Die Leitlinie der Deutschen Atemwegsliga und der Deutschen Gesellschaft für Pneumologie und Beatmungsmedizin nennt Ergotherapeuten jedoch explizit als Komponente der pneumologischen Rehabilitation [7].

Vor dem Hintergrund dieser Erkenntnisse haben sich die Autoren der Reporte dafür ausgesprochen, dass die Verordnungen dringend an den aktuellen wissenschaftlichen Kenntnisstand angepasst werden müssen - eine Chance für Ergotherapeuten, Patienten zukünftig so zu therapieren, wie es die aktuelle Evidenz empfiehlt.

\section{Mittler zwischen Forschung und Alltag >} Übrigens: Die Gründe für diese Ergebnisse kann qualitative Versorgungsforschung ans Licht bringen. Die Forscher können beispielsweise Interviews mit Ärzten führen und sie darin zu ihrem Verordnungsverhalten befra- gen. Möglich wäre auch, die Patienten zu interviewen, um zu erfahren, was sie tatsächlich brauchen.

Worin auch immer das Verordnungsverhalten der Ärzte begründet ist - die Analysen von Heil- und Hilfsmittelverordnungen machen unter anderem deutlich, dass evidenzbasierte Therapieansätze nicht automatisch ihren Weg in den Versorgungsalltag finden. Damit unterstreichen sie die Bedeutung der Versorgungsforschung als Mittler zwischen der klinischen Forschung und dem Versorgungsalltag. Und sie eröffnen vielfältige Forschungsmöglichkeiten für die Absolventen gesundheitsbezogener Studiengänge.

Eva Trompetter

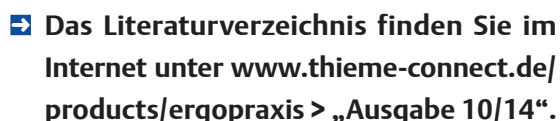
products/ergopraxis > „Ausgabe 10/14“.

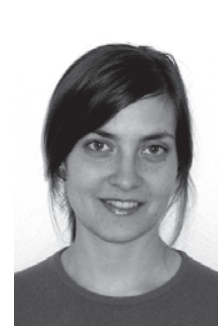

Eva Trompetter hat Public Health studiert und ist wissenschaftliche Mitarbeiterin an der FH Bielefeld. Sie schreibt regelmäßig für die Zeitschriften ergopraxis und physiopraxis. 